\title{
Actitudes y opiniones sobre el uso de prestaciones económicas por parte del colectivo inmigrante en el País Vasco
}

\author{
Gorka Moreno Márquez \\ Ikuspegi-Observatorio Vasco de Inmigración \\ Euskal Herriko Unibertsitatea-Universidad del País Vasco \\ <gorka.moreno@ehu.es>
}

\author{
Arkaitz Fullaondo Elordui-Zapaterietxe \\ Ikuspegi-Observatorio Vasco de Inmigración \\ Euskal Herriko Unibertsitatea-Universidad del País Vasco
}

\begin{abstract}
Artikulu honetan aztertzen da bertako jendearen jokabidea immigranteek osatutako kolektiboak egindako prestazio ekonomikoen erabileraren gaian. Zehazki, aztergai hartzen da diru-sarrerak bermatzeko errentaren gaia (lehenagoko oinarrizko errenta), hau da, Euskal Autonomia Erkidegoko diru-sarrerak bermatzeko politika publiko nagusia. Betekizun honetarako, xeheki analizatzen da urteroko Ikuspegi-Immigrazioaren Behatokiaren Atzerriko Immigrazioaren Jarrerak eta Pertzepzioen inguruko Barometroak 2010. eta 2011. urteetan buruturiko inkesta. Azterlanak erakusgai jartzen du biztanle immigranteek diru-sarrerak bermatzeko egiten duten erabileraz jokabide ezkorren nagusitzea. Fenomenoa interpretatu daiteke, neurri batean behintzat, egun bizi dugun krisi ekonomikoak baldintzatua, eta, ondorioz, immigrazioa mehatxu gisara nabarmentzen da.
\end{abstract}

\section{HITZ-GAKOAK:}

immigrazioa, gutxieneko errenta, jokabideak, gizarte-politikak, gizarte zaurgarritasuna.
Este artículo examina la actitud de la población autóctona hacia el uso de prestaciones económicas por parte del colectivo inmigrante. En concreto, hace especial hincapié en la renta de garantía de ingresos (antigua renta básica), la principal política pública en materia de garantía de ingresos en la Comunidad Autónoma del País Vasco. Para este cometido, se analiza detalladamente la información que ofrece al respecto el Barómetro anual sobre Percepciones y Actitudes hacia la Inmigración Extranjera en sus oleadas de 2010 y 2011, una encuesta que realiza Ikuspegi-Observatorio Vasco de Inmigración. El estudio pone de manifiesto el predominio de actitudes negativas hacia el uso que la población inmigrante hace de la renta de garantía de ingresos. Este fenómeno puede interpretarse, al menos en parte, como un reflejo de la actual crisis económica, que alimenta la percepción de la inmigración como una amenaza.

\section{Palabras clave:}

inmigración, rentas mínimas, actitudes, políticas sociales, vulnerabilidad social. 


\section{Introducción}

En este artículo vamos a indagar en el modo en que la población autóctona percibe el uso de la renta de garantía de ingresos, el programa de rentas mínimas de inserción existente en la Comunidad Autónoma del País Vasco (CAPV). Para ello, vamos a analizar los datos que aporta el Barómetro sobre Percepciones y Actitudes hacia la Inmigración Extranjera que realiza Ikuspegi-Observatorio Vasco de Inmigración. Se trata de una encuesta que sondea la opinión de la población autóctona hacia la inmigración en la CAPV. La primera oleada de este estudio se realizó en 2004 , y desde 2007 se ha elaborado con carácter anual. Son 1.200 encuestas y el margen de error se sitúa en un $\pm 2,82 \%$. El cuestionario es de carácter personal y se articula con base en un muestreo estratificado por cuotas, según el tamaño del hábitat, la edad y el sexo. Se compone de entre 25 y 30 preguntas -dependiendo del año-. En el caso concreto que atañe a nuestro artículo, desde la edición de 2010 se incluye un apartado compuesto por tres preguntas $(11,12 a$ y 12 b del cuestionario) sobre la percepción de la población autóctona acerca del uso de la renta de garantía de ingresos (RGI) por parte de la población inmigrante. Por ello, a lo largo de este trabajo vamos a analizar, en clave comparativa, los datos recogidos en estas preguntas a lo largo de los barómetros realizados en 2010 y 2011, haciendo especial hincapié en el más reciente.

El apartado sobre el uso de la RGI se ha introducido en las dos últimas ediciones del barómetro, porque es uno de los temas que ha suscitado mayor interés público y político en los últimos años cuando se analiza la actitud de la población autóctona hacia la inmigración en el País Vasco. El interés deriva de diferentes cuestiones. En primer lugar, y aunque el porcentaje de personas inmigrantes siga siendo relativamente bajo en la CAPV con respecto a otras comunidades autónomas -un 6,6\% frente al 12,2\% estatal-, en los últimos años se ha dado un cierto incremento del número de personas extranjeras en nuestro entorno, que ha hecho que poco a poco la inmigración haya ido ganando protagonismo tanto dentro de la opinión pública como de la agenda política.

En segundo lugar, la renta de garantía de ingresos es una prestación económica, asistencial, selectiva y no universal. Estas características la sitúan lejos de otras políticas sociales, como la sanidad o la educación, de carácter técnico o material, y universales, que son entendidas frecuentemente como un derecho humano universal. En la mayoría de los estudios realizados sobre percepciones hacia las políticas sociales, ya sean acotadas al colectivo inmigrante (Cea d'Ancona, 2007; Cea d'Ancona y Vallés, 2008, 2009, 2010; Rinken y Pérez Yruela, 2007; Rinken et al., 2009; Rinken, Escobar y Velasco, 2011; Ikuspegi, 2008, 2009) o a la población en general (Centro de Investigaciones Sociológicas, 2010; Gobierno Vasco, 2010), la tónica es que la aceptación de la sanidad o la educación es superior a la de otras políticas sociales, como, en nuestro caso, la RGI. Esta diferente valoración se da, en gran parte, debido a las características de unas y otra. En el caso del colectivo inmigrante, la percepción hacia esta ayuda por parte de la población autóctona es más negativa que hacia la sanidad o la educación.

En tercer lugar, las características y la magnitud de la renta de garantía de ingresos hacen de ella una prestación importante en la lucha contra la vulnerabilidad y la exclusión social, y que sea conocida por gran parte de la opinión pública. Este hecho, unido a sus condiciones -es un derecho subjetivo al que pueden acceder todas aquellas personas que lleven al menos un año empadronadas en la CAPV ${ }^{1}$, al margen de su nacionalidad o situación administrativa-, hacen que tanto el gasto en esta partida como su visibilidad social sean altos, y que el debate en torno a esta prestación sea notable, más aún en el caso del colectivo inmigrante.

Si a estos tres factores les sumamos la crisis económica y los recortes en materia social que estamos atravesando, y el aumento de personas que solicitan y acceden a esta prestación, tanto autóctonas como extranjeras, el contexto de competencia social es patente y tiene un claro reflejo en la controversia que se está dando acerca de su uso, muy en particular en lo que respecta al colectivo inmigrante. Por todo ello, creemos que resulta de gran utilidad profundizar en los datos que nos ofrece el barómetro y delimitar, en la medida de lo posible, ciertas afirmaciones y discursos que aparecen recurrentemente tanto en la opinión pública como en los medios de comunicación.

Antes de entrar en el análisis de los datos que emanan del cuestionario, definiremos y contextualizaremos la renta de garantía de ingresos de la CAPV, para así comprender mejor el contorno del debate y situar esta prestación dentro de la respuesta que se da, tanto a escala autonómica como estatal, a la vulnerabilidad y la exclusión social.

\section{La renta de garantía de ingresos y su contexto}

La renta de garantía de ingresos es el actual programa de rentas mínimas de inserción en el País Vasco. Dicho programa ha respondido a varias designaciones desde sus comienzos, allá por 1989. Inicialmente, fue conocido como ingreso mínimo familiar (IMF); entre 1990 y 2000 , se denominó ingreso mínimo de inserción (IMI); desde 2000 hasta 2008, renta básica; y tras la aprobación de la Ley para la Inclusión y la Garantía de Ingresos, en enero de 2009 , se llama renta de garantía de ingresos.

${ }^{1}$ Tras la reciente reforma realizada en la Ley de Inserción y Garantía de Ingresos de 2008, el requisito de un año de empadronamiento, a partir de enero de 2012, pasará a ser de tres años, o de uno más cinco de cotización en la Seguridad Social. 
Las rentas mínimas en el País Vasco nacen como una herramienta para hacer frente a la pobreza de finales de la década de 1980 , consecuencia principalmente de la crisis en el sector industrial y que irrumpe con gran fuerza en el debate político y social de aquellas fechas (Aguirre, 1991), tal como se puede comprobar en el informe La pobreza en la Comunidad Autónoma del País Vasco, publicado en 1987 (Gobierno Vasco, 1987). Con el paso de los años y los cambios sociales acaecidos en las últimas dos décadas, los programas de rentas mínimas vascos han ido centrándose en hacer frente a las problemáticas relacionadas con la vulnerabilidad y la exclusión social, de tal modo que hoy día la RGI es la principal herramienta de lucha contra la exclusión social en la CAPV (Moreno, 2008).

La renta de garantía de ingresos es un derecho subjetivo -y, por lo tanto, no atiende a limitación presupuestaria-, dirigido a aquellas personas que cumplen los requisitos que se enuncian a continuación:

- Existencia previa de una unidad de convivencia autónoma al menos un año antes de solicitar las prestación.

- Estar empadronado y residir en la CAPV al menos un año antes de solicitar la prestación (hasta diciembre de 2011) ${ }^{2}$.

- No poseer ingresos superiores a la cuantía establecida para cada caso y unidad de convivencia.

- Tener más de 23 años.

El aumento del número de personas perceptoras de la RGI ha sido sostenido en el tiempo. De las 4.110 personas que recibían el IMI en octubre de 1989 (Emaiker, s/a), se pasó a las 12.982 a finales de 2000 (Consejo Económico y Social Vasco, 2001: 425). Según los últimos datos disponibles ofrecidos por el Gobierno Vasco, en septiembre de 2011 eran 54.942 las personas titulares. El aumento en el número de las prestaciones, así como el incremento a lo largo de los años de la cuantía o una mayor flexibilidad en ciertos requisitos han hecho que el gasto en esta partida haya aumentado sensiblemente en estos últimos años. Así, dicho gasto era de 4,2 millones de euros en 1989, de 114 millones en 2004, y de 314 millones euros en 2010. La cuantía actual para una unidad de convivencia constituida por una sola persona es de unos 650 euros mensuales, que se reciben en 12 pagas anuales.

En lo tocante al perfil de las personas perceptoras, según una encuesta realizada a este colectivo (Gobierno Vasco, 2008), el 68,3\% son mujeres.

Ciñéndonos al tema que nos compete en este artículo y siguiendo con datos de esta encuesta, un $30 \%$ de las personas eran extranjeras, frente al $70 \%$ que eran autóctonas. Datos más recientes ofrecidos por Lanbide-Servicio Vasco de Empleo corroboran estas cifras y sitúan el número de mujeres beneficiarias

${ }^{2}$ Véase la nota anterior en un $65 \%$. También es destacable que un $27,4 \%$ sean personas mayores de 65 años. En lo tocante a la nacionalidad, los datos se mantienen estables: un $30,7 \%$ de extranjeros, frente a un $69,3 \%$ de autóctonos.

Todos estos datos se pueden entender mejor en el marco de los programas de rentas mínimas existentes a escala estatal, que, al ser de carácter autonómico, muestran una gran diversidad (Roberts, 2001; Gaviria y González, 2002). Una característica común es el escaso desarrollo de estos programas: con excepción de los programas vasco, navarro, madrileño o asturiano (Laparra y Ayala, 2009), en el resto de comunidades autónomas no puede hablarse realmente de programas de rentas mínimas. Miguel Laparra (2004b) subraya que, para el $80 \%$ de la población española, no existen programas de rentas mínimas.

Sobre todos los programas, destaca sobremanera el modelo vasco, que es el único que suele equipararse a otros similares del ámbito europeo (Moreno et al., 2003; Laparra, 2004a). Algunos ejemplos concretos pueden ilustrar esta afirmación. La renta de garantía de ingresos ofrece una cobertura seis veces superior a la media del resto de los programas de rentas mínimas estatales (Ministerio de Trabajo y Asuntos Sociales, 2005). De igual forma, según estimaciones de Laparra y Ayala (2009: 32) un tercio del total de los perceptores de rentas mínimas se sitúan en el País Vasco. Aunque no disponemos de datos concretos al respecto, los efectos de la crisis nos hace pensar que, debido a que la RGI es un derecho subjetivo en la CAPV, pero no en la mayoría de las comunidades autónomas, en torno al $50 \%$ del gasto total en rentas mínimas actuales puede circunscribirse al País Vasco. Estos datos nos dan una buena imagen de la dimensión del programa dentro del ámbito estatal y de su incidencia dentro del propio País Vasco.

En este contexto, se está dando una importante reflexión en torno a la renta de garantía de ingresos que, de forma más o menos directa, alcanza también al uso que de esta ayuda hace el colectivo inmigrante. Así, se espera que, para finales de 2011, se haya reformado en parte la Ley de Inclusión y Garantía de Ingresos (2008) -actualmente en tramitación legislativa-, que regula la ayuda que nos ocupa, y que la gestión de ésta pase de los servicios sociales de base a Lanbide-Servicio Vasco de Empleo. También se cree que puede haber modificaciones parciales en otros aspectos, en un contexto de contención presupuestaria, recortes en algunas prestaciones sociales, crisis económica y aumento exponencial del gasto en esta prestación en los últimos años ${ }^{3}$.

En este sentido se sitúa, precisamente, la propuesta lanzada por el Partido Popular del País Vasco días antes del inicio oficial de la campaña electoral de las

${ }^{3}$ N.E.: Este artículo se recibió a mediados de noviembre de 2011 $y$, por tanto, no tiene en cuenta los cambios legislativos producidos desde entonces. 
elecciones municipales (Vasco Press, 12-IV-11) de que el requisito de estar al menos un año empadronado en la CAPV aumente a cinco años. Parece que dicha propuesta podría llevarse al Parlamento e introducirse como enmienda en el nuevo texto legal en el que se está trabajando. Una medida de estas características afectaría, de facto, sobre todo al colectivo inmigrante, ya que todas las personas que hayan llegado recientemente -la mayoría, en el caso de los inmigrantes- se verían privadas de la posibilidad de solicitar la RGI.

En el plano social, también se detecta en los últimos meses el aumento de discursos y actitudes que relacionan al colectivo inmigrante con el fraude en la prestación de la renta de garantía de ingresos, o que apuntan a que este colectivo tiene mayores facilidades que otros a la hora de obtener dicha prestación u otras. En este sentido, puede destacarse la detección de estos discursos sobre todo en Internet a través de correos anónimos que inciden en este aspecto. También pueden encontrarse frecuentemente opiniones de este tipo en los comentarios que se recogen en las ediciones digitales de los principales periódicos de tirada regional, sobre todo en noticias referentes a la inmigración. El propio Ararteko (defensor del pueblo) ha hecho recientemente unas declaraciones que hacen hincapié en el riesgo de unir inmigración y uso fraudulento y abusivo de prestaciones sociales, así como en el aumento de este discurso dentro de la CAPV (Efe, 29-III-11).

Por todo ello, en el próximo apartado de este artículo vamos a intentar medir estas opiniones y así conocer mejor la situación, más allá de percepciones o discursos con mayor o menor incidencia mediática. El mismo Ararteko destacaba la inexistencia de estudios rigurosos al respecto y, en la medida de lo posible, este artículo quiere propiciar un mayor conocimiento dentro de este ámbito.

\section{La opinión de la población autóctona sobre el uso de la renta de garantía de ingresos por parte del colectivo inmigrante}

Como se apuntaba en la introducción, en este apartado vamos a analizar los datos referentes a la actitud de la población autóctona sobre el uso de la renta de garantía de ingresos por parte del colectivo inmigrante, tal como aparecen en el Barómetro anual sobre Percepciones y Actitudes hacia la Inmigración Extranjera. En concreto, examinaremos tres preguntas del cuestionario que hacen referencia directa a esa prestación. De igual forma, se contrastarán los datos de 2011 con los de 2010, buscando los cambios producidos de un año a otro.

En la primera de las cuestiones ( $n-11)$, se pregunta acerca de la postura que tendrían que tomar las instituciones con respecto al acceso de la RGI por parte de la población inmigrante. Un 6,9\% de las personas encuestadas opinan que tan sólo la deberían recibir las personas autóctonas y que las inmigrantes tendrían que quedar al margen. Un 31,2\% cree que los inmigrante la deberían recibir, pero únicamente cuando todos los autóctonos que la necesiten la hayan cobrado. Un $\mathbf{2 7 , 2} \%$ considera que tan sólo deben recibirla los inmigrantes en situación administrativa regular. Y finalmente, un $33,1 \%$ dice que la deberían obtener en las mismas condiciones que un autóctono -tal como sucede en la actualidad ${ }^{4}$-.

En esta pregunta no se aprecian grandes diferencias por sexo y edad, salvo por lo que se refiere a las personas de más de 65 años, que apoyan la preferencia de los autóctonos en mayor proporción que el resto. Según el nivel de estudios, puede subrayarse que aquellas personas con menor formación se muestran más restrictivas respecto al acceso. Así, un 47,3\% de la población sin estudios cree que los inmigrantes no deberían poder recibir la RGI (media: 31,2\%). Por el contrario, en ese grupo se recogen los menores apoyos (un $23 \%$ frente a una media del $33,1 \%$ ) a que la población inmigrante reciba esa ayuda en igualdad de condiciones. Aquellos con estudios universitarios o superiores, en cambio, muestran una pauta totalmente opuesta a los que no poseen estudios: son menos entre quienes niegan el acceso (un 12,2\%) y más (un $54,4 \%$ ) quienes abogan por la igualdad de condiciones de acceso.

Gráfico 1. Opinión de la ciudadanía sobre la postura que las instituciones deberían tomar con respecto a la renta básica, por nivel de estudios. CAPV, 2011 (\%)

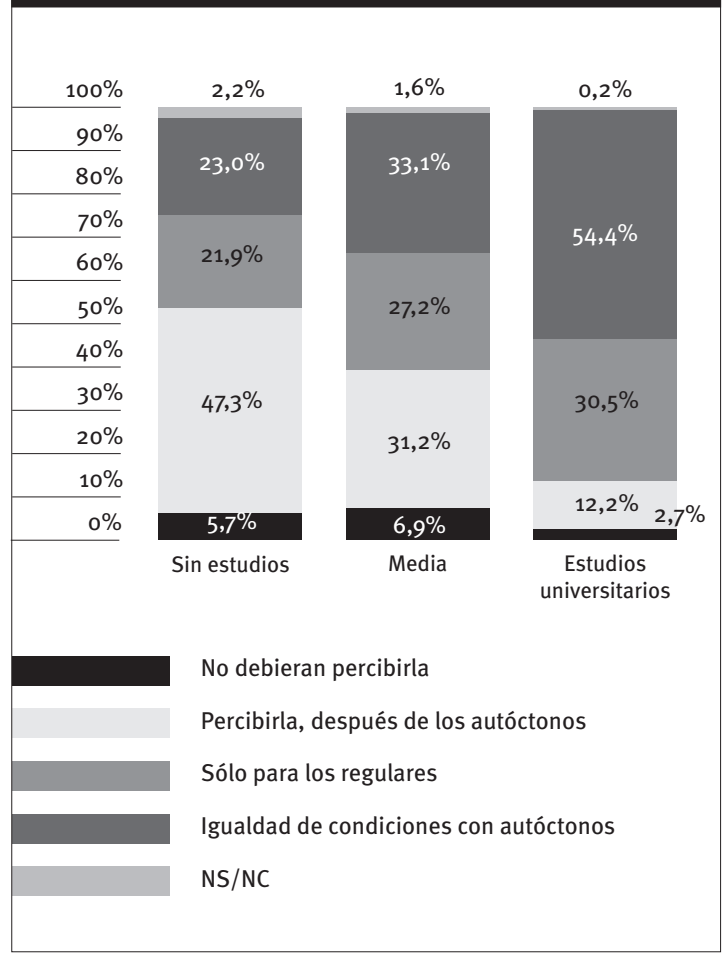

Fuente: Ikuspegi, Barómetro 2011.

${ }^{4}$ El trabajo de campo del barómetro 2011 se realizó entre marzo y abril de ese año. Por lo tanto, no se habían dado las recientes modificaciones normativas. 
Junto a los estudios, aquí toman relevancia las variables asociadas a la situación y la actividad económica. Así, entre las personas con mayores ingresos, es decir, quienes cobran entre 1.800 y 3.000 euros, y quienes cobran más de esta última cifra, la opción de que los inmigrantes accedan a la renta de garantía de ingresos es la mayoritaria: un $40,8 \%$ en el primer grupo y un $56,4 \%$ en el segundo. Estos porcentajes descienden al 34,8\% y al 31,4\%, respectivamente, entre aquellas personas con ingresos de hasta 600 euros, y de entre 600 y 1.800 euros.

En el caso de la actividad económica, mientras que los jubilados o las amas de casa muestran una actitud más reacia hacia el acceso a la prestación del colectivo inmigrante, las personas activas manifiestan una opinión más positiva. En este sentido, no podemos dejar a un lado que tanto las personas jubiladas como las amas de casa son frecuentemente colectivos precarios, con bajos ingresos, y además, en este caso concreto, perfiles habituales entre los perceptores de la renta de garantía de ingresos, tal como hemos podido observar cuando comentábamos brevemente los colectivos más representados entre los beneficiarios de esta prestación.

Por último, y para acabar con las variables asociadas a la situación socioeconómica, aquellas personas con un estatus social alto se muestran más receptivas hacia los inmigrantes, en oposición a aquellas que se sitúan en un estatus más bajo. Así, mientras que un $50,4 \%$ de las primeras creen que los inmigrantes tienen que acceder a la RGI en igualdad de condiciones, ese porcentaje desciende a un $22,8 \%$ entre aquellas que se sitúan en un estatus social bajo. Aunque el cambio en los enunciados de algunas de las respuestas de esta pregunta limitan en parte las posibles comparaciones, parece que los datos de 2011 son menos restrictivos que los de 2010. De este modo, el porcentaje de personas que creen que los inmigrantes no debían tener derecho a la prestación desciende de un $9,8 \%$ a un $6,9 \%$.

La segunda de las preguntas hace referencia al grado de acuerdo o desacuerdo en torno a la afirmación de que los inmigrantes se aprovechan excesivamente de la renta de garantía de ingresos y llegan, incluso, a acapararla. Un 33\% de las personas encuestadas están muy de acuerdo con esta afirmación, un 32,9\% están de acuerdo, un 10,5\% no están ni en acuerdo ni en desacuerdo, un 12,4\% están en desacuerdo, un $4,8 \%$ están muy en desacuerdo, y un $6,4 \%$ no saben o no contestan. Si aglutinamos las categorías que muestran acuerdo con la afirmación, podemos observar que casi dos tercios de las personas encuestadas $(65,9 \%)$ están de acuerdo con la idea de que los inmigrantes abusan de la RGI. En el lado opuesto, un $17,2 \%$ estaría en desacuerdo con dicha tesis.

En esta pregunta parecen percibirse las mismas pautas detectadas en la anterior. Así, entre quienes creen que los inmigrantes se aprovechan de la RGI se sitúan aquellas personas con más de 65 años, sin estudios o con estudios primarios, insatisfechas con su situación económica y con un estatus social bajo. En el lado opuesto, es decir, entre quienes más creen que los inmigrantes no abusan de la renta de garantía de ingresos, se encuentran las personas con estudios universitarios, agnósticas o ateas, con ingresos superiores a 3.000 euros y un estatus social medio-alto 0 alto.

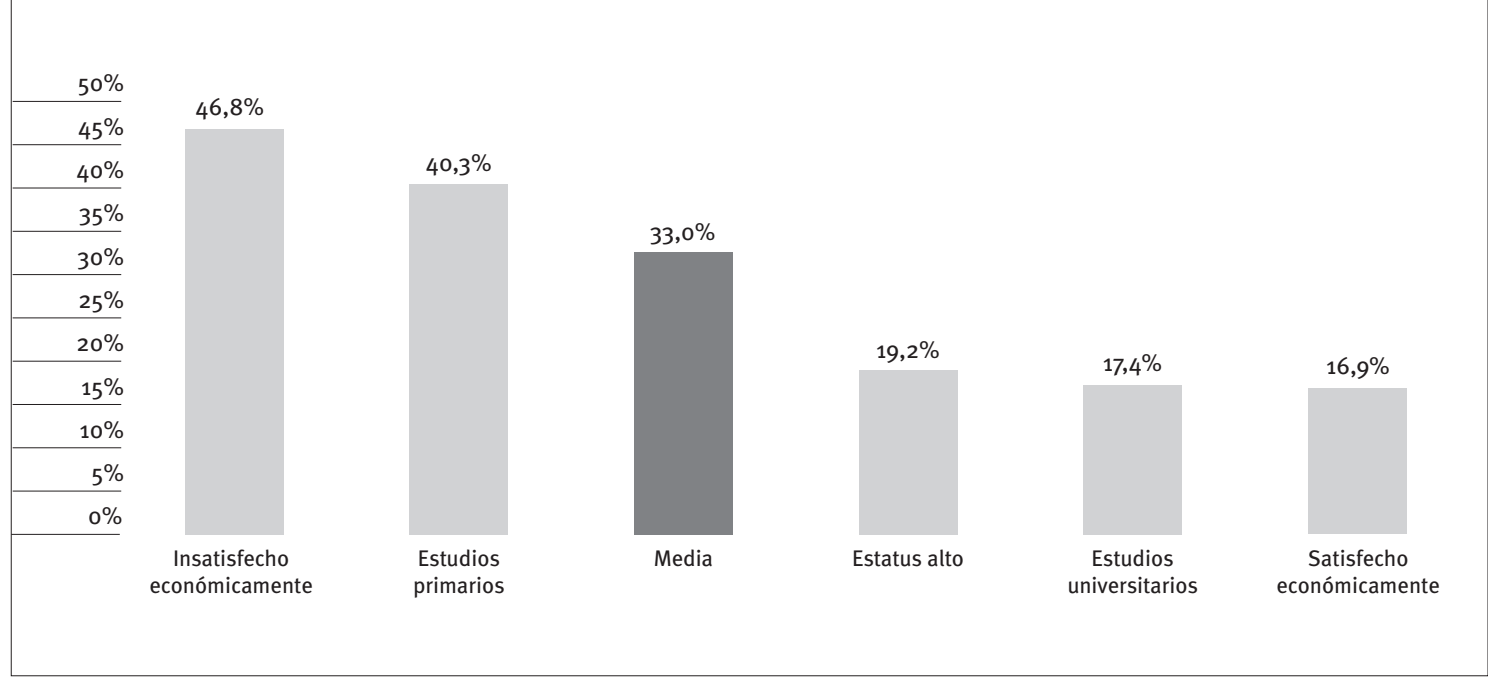

Fuente: Ikuspegi, Barómetro 2011. 
En términos comparativos, las cifras se mantienen muy similares a las del año anterior en todas las categorías, y tan sólo se detecta algún cambio, en especial en el nivel de intensidad. Así, aumenta el número de quienes están muy de acuerdo con la afirmación, y desciende el de quienes están de acuerdo. En el resto de opciones, no se detectan casi variaciones.

Tabla. 1. Opinión de la ciudadanía con la afirmación de que las personas inmigrantes se aprovechan excesivamente de la renta de garantía de ingresos, llegando a acapararla. CAPV, 2010 y 2011 (\%)

\begin{tabular}{l|c|c}
\hline & $\mathbf{2 0 1 0}$ & $\mathbf{2 0 1 1}$ \\
\hline Muy de acuerdo & 23,6 & 33,0 \\
\hline Acuerdo & 41,5 & 32,9 \\
\hline Acuerdo & 65,1 & 65,9 \\
\hline Ni acuerdo ni desacuerdo & 12,9 & 10,5 \\
\hline Desacuerdo & 12,8 & 12,4 \\
\hline Muy en desacuerdo & 4,1 & 4,8 \\
\hline Desacuerdo & 16,9 & 17,2 \\
\hline
\end{tabular}

Fuente: Ikuspegi, Barómetros 2010 y 2011.

Siguiendo con la vía abierta en la pregunta anterior, en la tercera y última pregunta del barómetro sobre el uso de la renta de garantía de ingresos por parte del colectivo inmigrante se ha sondeado el grado de acuerdo con la idea de que esta prestación hace que los inmigrantes no quieran trabajar o no busquen trabajo. Un 26,6\% está muy de acuerdo con esta afirmación; un 29,4\% está de acuerdo; un 17,3\%, ni de acuerdo ni en desacuerdo; un 15,8\% está en desacuerdo; y un 5,2\% está muy en desacuerdo. Como en la pregunta anterior, es mayoritaria la opinión de quienes consideran que el colectivo inmigrante hace un uso indebido de la RGI, aunque con un porcentaje algo menor, ya que un $56 \%$ cree que hace que no busquen un empleo o quieran trabajar, frente a un $21 \%$ que es contrario a esa idea.

En gran medida, se repiten las tendencias que han podido detectarse en las dos preguntas previas. Así, variables como los estudios, la religión, la ideología, la satisfacción económica, la actividad económica o el estatus marcan importantes diferencias en las respuestas. Aquellas personas con estudios universitarios, agnósticas o ateas, de izquierdas, con ingresos de más de 3.000 euros, satisfechas económicamente, estudiantes o de estatus alto son más reacias a creer que la RGI desincentiva la búsqueda de un empleo en el colectivo inmigrante, mientras que aquellas personas con estudios primarios, de derechas, insatisfechas económicamente o en paro muestran un mayor grado de acuerdo con esa afirmación.

Con respecto al año anterior, se detectan dos pautas que aparecen por separado en las dos preguntas previas. Por un lado, las respuestas más positivas hacia la inmigración son mayores, en la línea de los datos globales del Barómetro 2011, que son mejores que los del año anterior en términos generales. De esta forma, aquellas personas que están muy en desacuerdo con la afirmación de que la RGI desincentiva el empleo de los inmigrantes sumaban un 3,3\% en 2010 , y son un $5,2 \%$ en 2011 . De igual forma, aquellos que están en desacuerdo con esta afirmación pasan de un $13,8 \%$ a un $15,8 \%$. Agrupando estas dos categorías, aquellas personas en desacuerdo eran un $17,1 \%$ el año pasado y son un $21 \%$ este año. Por otro lado, aunque desciende el porcentaje de quienes están de acuerdo con la afirmación -en 2010 eran un $59,7 \%$ y en el 2011 son un $56 \%$-, el cambio más destacable es en la intensidad, ya que las opiniones negativas más extremas hacia el colectivo inmigrante toman fuerza ( $26,6 \%$ en 2011 frente a $19,6 \%$ en 2010), en detrimento de las más moderadas (29,4\% en 2011 y $40,1 \%$ en 2010).

Tabla 2. Opinión de la ciudadanía sobre la afirmación de que la renta básica hace que inmigrantes no quieran trabajar, o no busquen trabajo. CAPV, 2011 (\%)

\begin{tabular}{|c|c|c|c|c|c|c|c|}
\hline & \multirow{2}{*}{ Media } & \multicolumn{2}{|c|}{ Estudios } & \multicolumn{2}{|c|}{ Ideología } & \multicolumn{2}{|c|}{ Satisfacción económica } \\
\hline & & Primarios & Universitarios & Derecha & Izquierda & Insatifecho & Satisfecho \\
\hline Muy de acuerdo & 26,6 & 32,7 & 16,0 & 29,9 & 23,6 & 35,9 & 12,8 \\
\hline Acuerdo & 29,4 & 32,4 & 20,7 & 43,6 & 18,8 & 23,0 & 31,0 \\
\hline Acuerdo & 56,0 & 65,1 & 36,7 & 73,5 & 42,4 & 58,9 & 43,8 \\
\hline $\mathrm{Ni}$ acuerdo ni desacuerdo & 17,3 & 16,3 & 16,9 & 13,5 & 21,7 & 16,1 & 18,7 \\
\hline Desacuerdo & 15,8 & 10,9 & 23,8 & 8,0 & 23,2 & 19,9 & 23,8 \\
\hline Muy en desacuerdo & 5,2 & 2,1 & 15,1 & 0,0 & 11,9 & 3,6 & 5,4 \\
\hline Desacuerdo & 21,0 & 13,0 & 38,9 & 8,0 & 35,1 & 23,5 & 29,2 \\
\hline
\end{tabular}

Fuente: Ikuspegi, Barómetro 2011. 


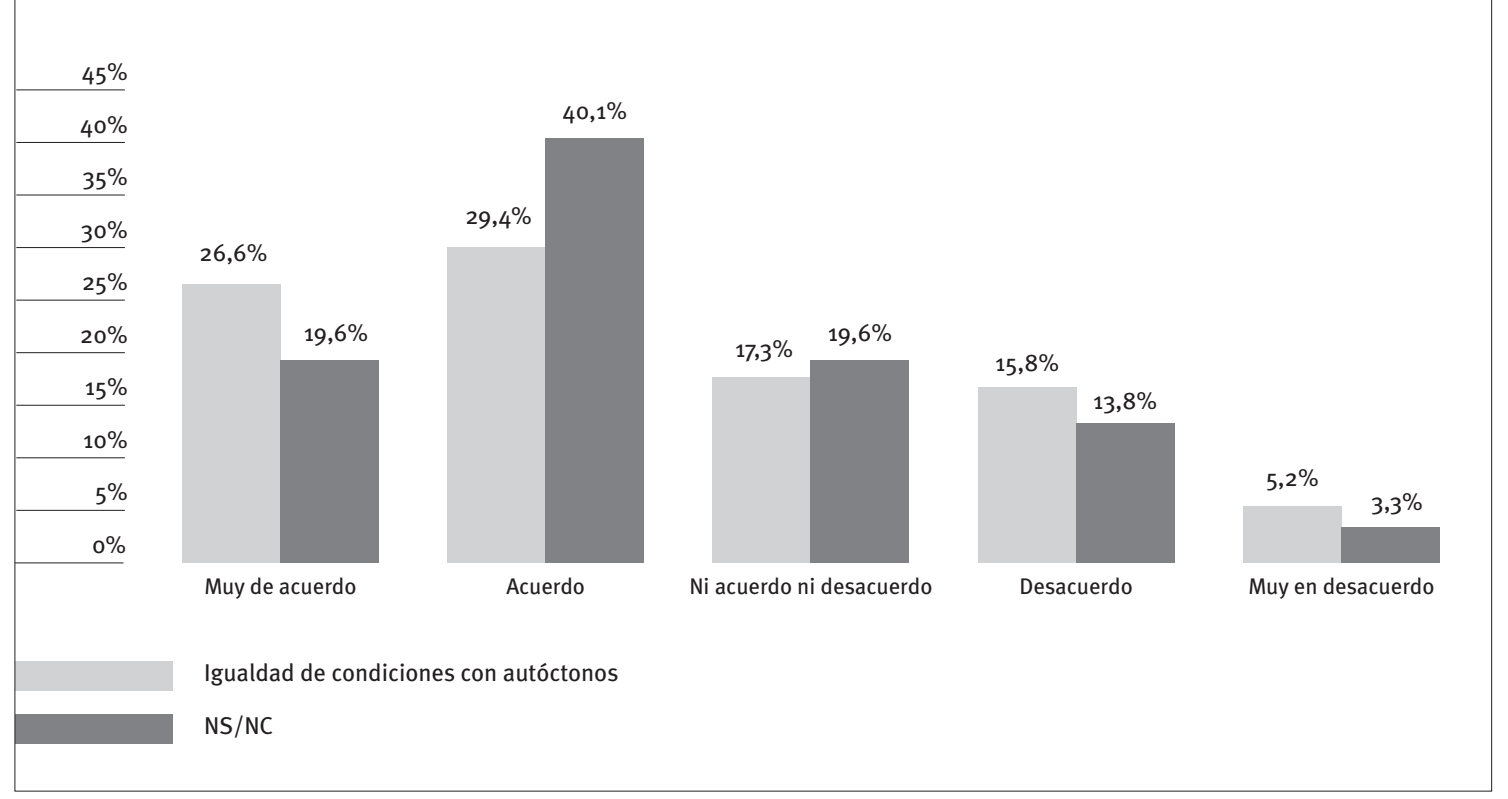

Fuente: Ikuspegi, Barómetro 2010 y 2011.

\section{Conclusiones}

Para acabar este artículo, queremos compilar algunos de los resultados más relevantes sobre los datos presentados en el punto anterior y plantear también ciertas reflexiones que sirvan para el debate. En primer lugar, hay que destacar que las actitudes hacia el uso de la renta de garantía de ingresos por parte del colectivo inmigrante son mayoritariamente negativas, y en todas las preguntas realizadas, prevalecen las opiniones que subrayan el mal uso o los efectos negativos de la prestación sobre este colectivo. Únicamente un tercio de las personas encuestadas $(33,1 \%)$ se posicionan a favor de los requisitos actuales, en los que prevalece la igualdad de condiciones en el acceso tanto para autóctonos como para extranjeros. Aunque tan sólo un $6,9 \%$ cree que no deberían cobrarlo, un 65,3\% opta por una gestión más restrictiva hacia el colectivo inmigrante. En las otras respuestas también prevalecen las respuestas negativas. Así, un $65,9 \%$ opina que los inmigrantes abusan de la RGI, y un 56\%, que desincentiva la búsqueda de empleo. Las posiciones contrarias a esta afirmación se sitúan, respectivamente, en un $17,2 \%$ y en un $21 \%$.

En lo tocante a las diferencias entre los datos de 2010 y 2011, cabe subrayar que se percibe una actitud más favorable al colectivo inmigrante en 2011, una pauta que ha podido detectarse en otras cuestiones abordadas en el Barómetro 2011. De igual modo, también parece detectarse que, aunque las opiniones más negativas no aumentan, la intensidad de éstas sí que lo hace, de tal manera que las opiniones negativas más moderadas pierden fuerza en detrimento de las más extremas. Habrá que comprobar si en próximas ediciones del Barómetro, y más aún en un contexto de crisis económica y competencia por los recursos sociales existentes, esta tendencia se afianza o aumenta, o si se trata únicamente de una pauta coyuntural o temporal.

Por colectivos o variables, puede detectarse una serie de diferencias significativas que merece la pena destacar. En este sentido, y siguiendo la pauta general del Barómetro, aquellas personas con menores estudios, en situación de inactividad económica, con bajos ingresos, insatisfechos económicamente o con un estatus social más bajo muestran opiniones más negativas hacia la inmigración en general, y hacia el uso que hacen de la RGI en concreto. En el lado opuesto, se sitúan aquellas personas con estudios universitarios, activas o estudiantes, con ingresos altos, satisfechos económicamente, con un estatus social alto, agnósticas o ateas, y de izquierdas.

En todo caso, son las variables socioeconómicas las que más diferencias muestran y las que parecen explicar estas diferencias en mayor medida. Así, los estudios, la satisfacción económica o el estatus social marcan diferencias mucho más notorias que otras variables. La variable ingresos mostraba en 2010 una influencia mayor que en 2011, aunque sigue arrojando importantes diferencias.

Aquellas personas con menores ingresos, mayor insatisfacción económica y menor estatus social muestran una opinión más desfavorable al uso de la RGI por parte del colectivo inmigrante; $y$, a la inversa, aquellas personas con mayores ingresos muestran una opinión más positiva, o por lo menos más 
moderada y atemperada. Este patrón se repite en la mayoría de las encuestas realizadas sobre opiniones y actitudes en torno a la inmigración -ya citadas en este artículo- y en las diferentes ediciones del Barómetro de Ikuspegi-Observatorio Vasco de Inmigración también ha aparecido recurrentemente.

De esta forma, aquellos colectivos con menos seguridad vital, social y económica son los que se muestran más reacios a la utilización de la renta de garantía de ingresos por parte del colectivo inmigrante. De hecho, las preguntas plantean afirmaciones clásicas en torno a este tipo de prestaciones económicas, que históricamente han tenido cierta relevancia en la opinión pública, sobre todo cuando se relacionan con colectivos autóctonos precarios o en situación de exclusión social, como el gitano. En la actualidad, resulta destacable cómo estas mismas afirmaciones son empleadas por las clases bajas autóctonas para denunciar el uso de estas prestaciones por parte de los inmigrantes. No es nada nada extraño, ya que son los grupos autóctonos peor situados los que compiten directamente con gran parte de los inmigrantes para acceder a los nichos laborales más precarios, o a las prestaciones de carácter asistencial que se dirigen a hacer frente a la vulnerabilidad y la exclusión social.

En el lado opuesto de la estructura social, se sitúan aquellas personas con ingresos altos, satisfechas económicamente o con un estatus social alto. Es decir, las clases medias, que muestran un discurso mucho más amable hacia la inmigración en general.
En este sentido, no podemos obviar que, para este sector, la población inmigrante no suele suponer una competencia, ya que no se sitúan en el mismo escalafón laboral o de acceso a prestaciones sociales. Es más, en muchas ocasiones la inmigración se convierte, para las clases medias, en una suerte de ventaja añadida, ya que, gracias a ella, pueden cubrir necesidades relacionadas con los cuidados domésticos y personales que facilitan un mejor acceso al mercado laboral, o una mayor disponibilidad horaria para el ocio.

Como puede observarse, el contexto dual y sin vasos comunicantes que surge en la relación entre los colectivos autóctonos y el inmigrante propicia una dinámica en la que es muy lógico que unos vean la inmigración como una amenaza, mientras que otros la ven como una oportunidad. Es un escenario en el que se permite la construcción de discursos contrarios y negativos hacia la inmigración, por un lado y, discursos favorables y positivos por el otro. Más aún, la crisis que estamos viviendo alimenta estos estados de opinión y es el contexto más propicio para que puedan reproducirse lo que en palabras de De la Corte y Blanco son el "discurso impecable e implacable” hacia la inmigración (2006). Resulta de gran interés hacer un seguimiento de estas actitudes en los próximos años, condicionados por los efectos de la crisis, para ver si la opinión sobre el uso de las prestaciones económicas por parte del colectivo inmigrante aumenta o se mantiene en cifras similares a las actuales. 
AGUIRRE, J. A. (1991): “Evaluación del primer año de implantación del ingreso mínimo familiar en el País Vasco", en VV.AA., IV. Jornadas de Economía de los Servicios Sociales, VitoriaGasteiz, Gobierno Vasco.

CEA D’ANCONA, M. A. (2007): Inmigración, racismo y xenofobia en la España del nuevo contexto europeo, Madrid, Observatorio Español del Racismo y la Xenofobia.

CEA D’ANCONA, M. A.; y VALLES, M. S. (2010): Evolución del racismo y la xenofobia en España. Informe 2010, Madrid, Observatorio Español del Racismo y la Xenofobia [khttp://www.oberaxe. es/files/datos/4e20230088dc8/INFORME\%20 RACISMO\%202010.pdf >].

- (2009): Evolución del racismo y la xenofobia en España. Informe 2009, Madrid, Observatorio Español del Racismo y la Xenofobia [<http://www. oberaxe.es/files/datos/4b26574eb2f66/ Informe2009.pdf $\rangle$.

- (2008): Evolución del racismo y la xenofobia en España. Informe 2008, Madrid, Observatorio Español del Racismo y la Xenofobia [<http://www. oberaxe.es/files/datos/49dgb81191e7f/ CEA2008.pdf $>$.

CENTRO DE INVESTIGACIONES SOCIOLÓGICAS (2010): Opinión pública y política fiscal, serie Estudios, no 2.841, Madrid, Centro de Investigaciones Sociológicas [khttp://www.cis.es/cis/opencm/ ES/1_encuestas/estudios/ver.jsp?estudio $=105$ $62 \&$ cuestionario $=12616 \&$ muestra $=18139$ > $]$.

CONSEJO ECONÓMICO Y SOCIAL VASCO (2001): Memoria socioeconómica 200o: Comunidad Autónoma del País Vasco, Bilbao, Consejo Económico y Social Vasco, pág. 425.
DE LA CORTE, L.; y BLANCO, A. (2006): “Conflictos intergrupales y prejuicios étnicos en las sociedades receptoras de inmigrantes", en VV.AA., Inmigración. Un desafío para España, Madrid, Fundación Pablo Iglesias.

EFE (2011) “El Ararteko ve 'alarmante’ unir inmigración y prestaciones sociales", El Correo, 29-III-11 [rhttp://www.elcorreo.com/alava/20110329/ mas-actualidad/sociedad/ararteko-alarmanteunir-inmigracion-201103291237.html/].

EMAIKER (s/d): Informe del estudio-panel de beneficiarios del ingreso mínimo de inserción, VitoriaGasteiz, Eusko Jaurlaritza-Gobierno Vasco.

GAVIRIA, L.; y GONZÁLEZ, F. (2002): Propuestas integradas de políticas activas de bienestar y empleo: sumario, Dublín, Fundación Europea para la Mejora de las Condiciones de Vida y de Trabajo [khttp://www.eurofound.europa.eu/ pubdocs/2002/60/es/1/efo26oes.pdf>].

GOBIERNO VASCO (2010): Políticas públicas, serie Sociómetro Vasco, no 43, Vitoria-Gasteiz, Eusko Jaurlaritza-Gobierno Vasco.

- (2008): Estudio de las personas perceptoras de Renta Básica, Vitoria-Gasteiz, Eusko JaurlaritzaGobierno Vasco.

- (1987): La pobreza en la Comunidad Autónoma del País Vasco, Vitoria-Gasteiz, Eusko JaurlaritzaGobierno Vasco.

IKUSPEGI (2011): Barómetro 2010. Percepciones y actitudes hacia la inmigración extranjera, Bilbao, Ikuspegi [khttp://www.ikuspegi-inmigracion. net/documentos/barometros/2010/ barometro2010cas.pdf)].

- (2010): Barómetro 2009. Percepciones y actitudes hacia la inmigración extranjera, Bilbao, Ikuspegi [khttp://www.ikuspegi-inmigracion.net/ 
documentos/investigacion/es/bar_2009_cas. pdf>].

- (2008): Barómetro 2007. Percepciones y actitudes hacia la inmigración extranjera. Bilbao, Ikuspegi [«http://www.ikuspegi-inmigracion.net/ documentos/investigacion/es/bar_2008_ completo.pdf〉].

LAPARRA, M. (2004a): "La travesía del desierto de las rentas mínimas en España”, Documentación Social, no 135, págs. 57-76 [<http://www.caritas.es/ imagesrepository/CapitulosPublicaciones/485/ Doc.\%20Social\%20135\%20Capitulo\%203. pdf>].

- (2004b): “Las rentas mínimas en España”. Trabajo presentado en el 'Encuentro Renta Básica y Procesos de Inserción, 1-2 diciembre, VitoriaGasteiz'.

LAPARRA, M.; y AYALA, L. (2009): El sistema de garantía de ingresos mínimos en España y la respuesta urgente que requiere la crisis social, Madrid, Fundación FOESSA [<http://www.caritashuelva. org/spip212/IMG/pdf_SGIMEspana2009.pdf〉].

MINISTERIO DE TRABAJO Y ASUNTOS SOCIALES (2005): III Plan Nacional de Acción para la Inclusión Social del Reino de España. 2005-2006, Madrid, Ministerio de Trabajo y Asuntos Sociales [rhttp://www.mtas.es/SGAS/ServiciosSocDep/ inclusionsocial/plannacional/IIIPNAin.pdf〉].

MORENO, G. (2008): Crisis y reconstrucción del Estado del bienestar. Las posibilidades de la Renta Básica de Ciudadanía, Vitoria-Gasteiz, Gobierno Vasco.

MORENO, G.; y AIERDI, X. (2008): “Inmigración y servicios sociales: ¿última red o primer trampolín?”, Zerbitzuan, no ${ }^{44}$, diciembre, págs. 7-18 [rhttp://www.zerbitzuan.net/documentos/ zerbitzuan/Inmigracion $\% 20 y \% 20$ servicios\%20 sociales.pdf $\rangle]$.
MORENO, L.; et al. (2003): "Existe una malla de seguridad en la Europa del sur", serie Documentos de Trabajo, n-0 03-17, Consejo Superior de Investigaciones Científicas [rhttp://hdl.handle. net/10261/1579>].

RINKEN, S.; ESCOBAR, Mํ. S.; y VELASCO, S. (2011): Opiniones y actitudes de la población andaluza ante la inmigración (III): más allá del discurso funcionalista, Sevilla, Junta de Andalucía [<http://www.juntadeandalucia.es/empleo/ www/adjuntos/publicaciones/1_2144_LibroOPIA-III.pdf>].

RINKEN, S; et al. (2009): Opiniones y actitudes de los andaluces ante la inmigración (II): entre la estabilidad y el cambio, Sevilla, Junta de Andalucía [shttp://www.juntadeandalucia. es/empleo/opam/sites/default/files/DOC/ texto_completo_OPIA_II.pdf \].

RINKEN, S.; y PÉREZ YRUELA, M. (2007): Opiniones y actitudes de la población andaluza ante la inmigración, Sevilla, Junta de Andalucía [<http://digital.csic.es/ bitstream/10261/15129/1/OPAM_7.pdf>].

ROBERTS, E. (2001): “Coordination of activation programmes for minimum income recipients. European experiences: A synthesis". Comunicación presentada en la $5^{\text {th }}$ Conference 'Visions and Divisions. Helsinki, August 28 - September 1, 2001', organizada por la European Sociological Association [khttp:// www.shakti.uniurb.it/Eurex/esa/PDF/ESARoberts.pdf $\rangle$.

VASCO PRESS (2011) “Basagoiti propone pedir cinco años de empadronamiento para percibir ayudas sociales", El Correo, 12-IV-11 [shttp://www. elcorreo.com/alava/20110412/local/basagoitipropone-pedir-cinco-201104121316.html ]. 